\title{
Mesoamerican Nephropathy (MeN): What We Know so Far
}

\author{
Vicente Sanchez Polo (1D) \\ Ramon Garcia- \\ Trabanino (1D) 2,3 \\ Guillermo Rodriguez ${ }^{4}$ \\ Magdalena Madero ${ }^{5}$ \\ 'Instituto Guatemalteco de Seguridad \\ Social, Guatemala, Guatemala; ${ }^{2}$ Centro \\ de Hemodiálisis, San Salvador, El \\ Salvador; ${ }^{3}$ Fondo Social de Emergencia \\ Para la Salud, Tierra Blanca, El Salvador; \\ ${ }^{4}$ Service of Nephrology, Hospital Dr. R.A. \\ Calderón Guardia, Caja Costarricense de \\ Seguro Social, San José, Costa Rica; \\ ${ }^{5}$ Division of Nephrology, Instituto \\ Nacional de Cardiología Ignacio Chávez, \\ México, México
}

This article was published in the following Dove Press journal: International Journal of Nephrology and Renovascular Disease

\begin{abstract}
In 2002, a report from El Salvador described a high incidence of chronic kidney disease (CKD) of unknown cause, mostly in young males from specific coastal areas. Similar situations were observed along the Pacific Ocean coastline of other Central American countries and southern Mexico (Mesoamerica). This new form of CKD has been denominated Mesoamerican endemic nephropathy $(\mathrm{MeN})$. The typical presentation of $\mathrm{MeN}$ is a young male from an endemic area with a family history of CKD, low eGFR, high serum creatinine, low level of albuminuria, hypokalemia, hyperuricemia, and urine urate crystals. Kidney biopsy demonstrating tubulointerstitial nephritis remains the gold standard for diagnosis but is available only for a minority. Commonly proposed causes include thermal stress/dehydration and/or exposure to environmental pollutants. However, likely, a third factor, which could be genetic or epigenetic, could contribute to the cause and development of the disease, along with social determinants. Currently, preventive measures focus on minimizing workers exposure to thermal stress/dehydration. There are many research opportunities and priorities should include clinical trials to evaluate the efficacy and safety of the current treatment protocols, along with etiological and genetic studies, and the development of kidney disease data systems. Although there is scant and controversial literature with regard $\mathbf{s}$ to the etiology, diagnosis and management of the disease, our aim is to provide the reader a vision of the disease based on our experience.
\end{abstract}

Keywords: Mesoamerican nephropathy, MeN, heat stress, CKDu, CKDnt, endemic nephropathy, regional nephropathy

\section{Mesoamerican Nephropathy: From Theory to Bedside Introduction}

There are some specific regions of the world where new forms of Chronic Kidney Disease (CKD), not explained by the traditional risk factors such as diabetes and hypertension, have been identified and described during the last two decades. These regional endemic nephropathies are generically called CKD of undetermined cause $(\mathrm{CKDu})$ or $\mathrm{CKD}$ of non-traditional etiology (CKDnt), and more recently, chronic interstitial nephritis of agricultural communities (CINAC), but most often locally identified by its geographical location, eg.; Sri Lanka nephropathy, Uddanam nephropathy and Mesoamerican endemic nephropathy (MeN). ${ }^{1-8}$

Typically, MeN is present in young male inhabitants from low income, rural populations located in the warm zones of the Pacific Ocean coastline of southern Mexico and Central America - hence the geographic designation. Strenuous working
Correspondence: Magdalena Madero Division of Nephrology, Instituto Nacional de Cardiologia Ignacio Chavez, Juan Badiano I, Col Seccion XVI, México CP 14080, México

Email madero.magdalena@gmail.com
International Journal of Nephrology and Renovascular Disease 2020:13 26I-272 
conditions, exposure to high heat stress, environmental pollutants and agrochemicals, frequent use of self-prescribed painkillers and dehydration are features commonly described among these populations, besides relevant social determinants as poverty, inadequate access to healthcare, malnutrition, low birth weight and high rates of social violence. ${ }^{8} \mathrm{MeN}$ is one of the conditions which are overrepresented in disadvantaged populations. Apart from being more frequent, kidney disease among disadvantaged populations tends to be underdiagnosed. When detected, access to care is limited and, frequently, sub-optimal. Irrespective of the underlying cause of CKD, the combination of these two factors - under-diagnosis and sub-optimal treatment contributes to poor clinical outcomes. Moreover, the development of end-stage kidney disease (ESKD) in communities in which access to kidney replacement therapy (KRT) is limited or non-existent forces households to incur substantial "out of pocket" expenses, perpetuating the poverty cycle. ${ }^{9-13}$ Illustrating this, the average annual CKD mortality rate reported for adult males at the region of the Bajo Lempa in El Salvador, an endemic hotspot of $\mathrm{MeN}$, is as high as 387 per 100,000 inhabitants, up to 30 times higher than comparable countries in the Americas. ${ }^{14}$ Unfortunately, none of the implicated countries has kidney disease surveillance systems in place; hence, the real burden of disease is as undetermined as its cause.

This review is written by frontline clinical nephrologists and researchers from the Mesoamerican region and thus is focused on MeN, aiming to review and update the published knowledge about the disease, but mostly to provide clinical insights that may contribute to a better understanding of the disease. Although there is scant and controversial literature with regard to the etiology, diagnosis and management of the disease, our aim is to provide the reader a vision of the disease based on our regional experience.

\section{Timeline and Presentation}

It is difficult to determine how long these hotspots have existed, partly due to the absence of systematic records, and partly due to the poverty of the communities affected. Furthermore, nephrology did not develop as a field of practice in most Central American countries until the last decades. In addition, the clinical work demand is high, and there are little or no funding opportunities to support research. In El Salvador, the high incidence of young male CKD patients of undetermined cause presenting at the emergency wards was considered "normal" until it was formally reported by the end of the last century, which allowed for comparisons that made apparent this was an atypical presentation. ${ }^{15}$ Likewise, in Nicaragua, many local studies, mainly from the National Autonomous University of Nicaragua in Leon (UNAN Leon) and Ingenio San Antonio, reflected the curiosity of colleagues and students who had been observing in daily practice for years an intriguingly high prevalence of mortality from CKD in young male adults from the lowlands in northwestern Nicaragua. Some of these local reports show data going back as far as the late 1970s. ${ }^{16}$ In Costa Rica, a more recent retrospective study showed a similar situation, high mortality from CKD in young male adults from the lowlands in north-western Costa Rica at least from the '70s. ${ }^{17-19}$ Due to the absence of official records and inadequate access to nephrology health care it took a long time for health authorities to acknowledge the existence of a problem that was obvious to the local communities (Figure 1).

The typical clinical presentation for $\mathrm{MeN}$ is a young male from an endemic coastal hotspot with a family history of CKD. To date, many patients still present through the emergency ward with End-Stage Kidney Disease (ESKD) requiring urgent dialysis initiation. Nevertheless, thanks to increasing awareness, early diagnosis has also improved in some regions and many patients are being diagnosed at earlier stages of CKD through community screening campaigns. Others seen before Kidney replacement therapy (KRT) initiation seek medical attention for symptoms such as a sensation of "fever", aseptic dysuria, nocturia, and cramps and/or muscular weakness in association with electrolyte abnormalities. Acute Kidney Injury (AKI) is often a manifestation of the disease. Family history of CKD is common and past medical history is usually nonrelevant. On physical exam, patients are usually normotensive; signs of fluid overload are usually absent. In some cases, blood pressure may be high, especially when the disease occurs at the later stages. Common laboratory findings include hyperuricemia, hypokalemia, hyponatremia, low-grade non-nephrotic proteinuria, and urine analysis can show isosthenuria and urate crystals. When performed, ultrasound at early stages of CKD demonstrates normal-sized kidneys and increased echogenicity of the parenchyma, with no evidence of obstruction or hydronephrosis. ${ }^{5,22-24}$ Progression from initial onset to CKD and later to ESKD can be quick in some patients and even more so if not treated timely. ${ }^{25,26} \mathrm{It}$ is important to emphasize that any $\mathrm{CKD}$ will evolve more 
Mesoamerican nephropathy: early timeline.

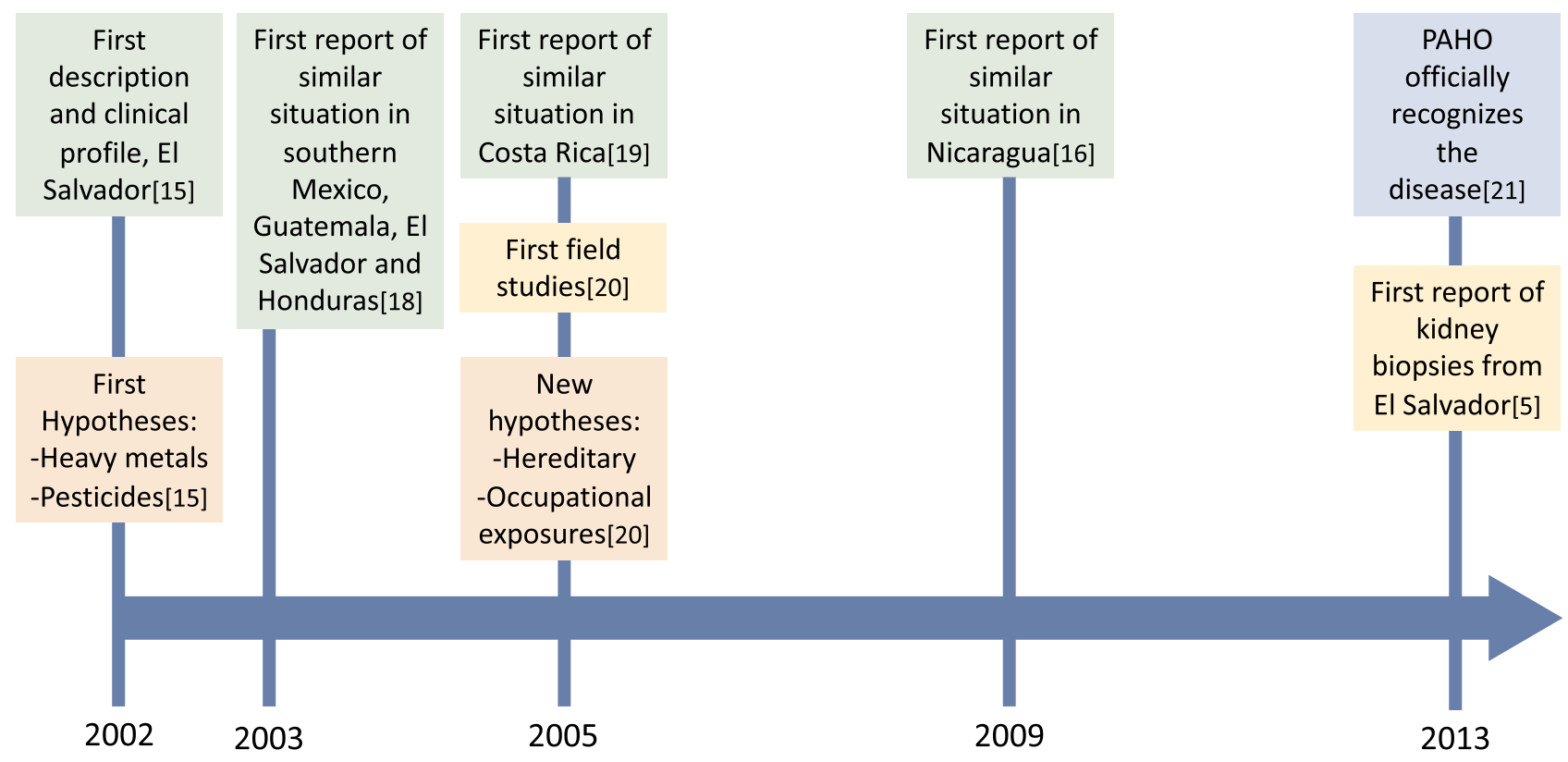

PAHO: Pan American Health Organization

Figure I Mesoamerican Nephropathy: Early timeline. During the first years after the initial description of the disease, the progress of knowledge was slow, mainly due to the plight of the developing countries where it occurs.

favorably under timely, proper nephrology care, and $\mathrm{MeN}$ should not be an exception. Figure 2 summarizes the patients' clinical presentation and evolution.

\section{Definition of Suspected and Confirmed Case}

One of the most important challenges in CKDu has relied on the agreement on a universal definition. The heterogenicity of the CKDu definition has been part of the obstacle for preventing and treating the disease. A global and uniform approach to detect and diagnose CKDu is urgently needed to establish comparisons between different regions and studies. The International Society of Nephrology has worked on a Consortium of Collaborators in order to guide a common approach to the detection of CKDu by working on a minimum dataset. ${ }^{27}$ The gold standard definition for CKDu has relied on the histopathological confirmation of the disease; however, kidney biopsy is not feasible for most patients, and therefore we need to accept a certain level of uncertainty.

The definition of CKDu can be divided into suspicious, probable, and confirmed cases based on the different levels of data obtained. Although the KDIGO definition of CKD requires two-measurements of eGFR, this is cumbersome, and based on a large body of CKD epidemiology, we recommend accepting the initial detection based on a single eGFR determination. ${ }^{27}$ Table 1 describes the required criteria for suspected, probable and confirmed CKDu.

\section{Risk Factors and Pathophysiology}

The risk factors and etiology of MeN are highly controversial, current evidence is limited and at this point may help with hypothesis generation and future study design. In the next paragraphs, we will discuss the most accepted theories to explain the disease (Figure 3).

\section{Heat Stress and Dehydration}

Populations mainly affected by MeN live at altitudes below 300 meters above sea level and for the most part work in agriculture. ${ }^{20-24}$ Other areas in Mexico and Central America have been identified as hot spots. More recently, Aguilar-Ramirez et al described another probable hotspot in Veracruz, Mexico, an area that shares the same demographic characteristics and agriculture as the main occupation. ${ }^{29}$ Since the first described area, multiple 


\section{Mesoamerican nephropathy: clinical presentation and evolution.}

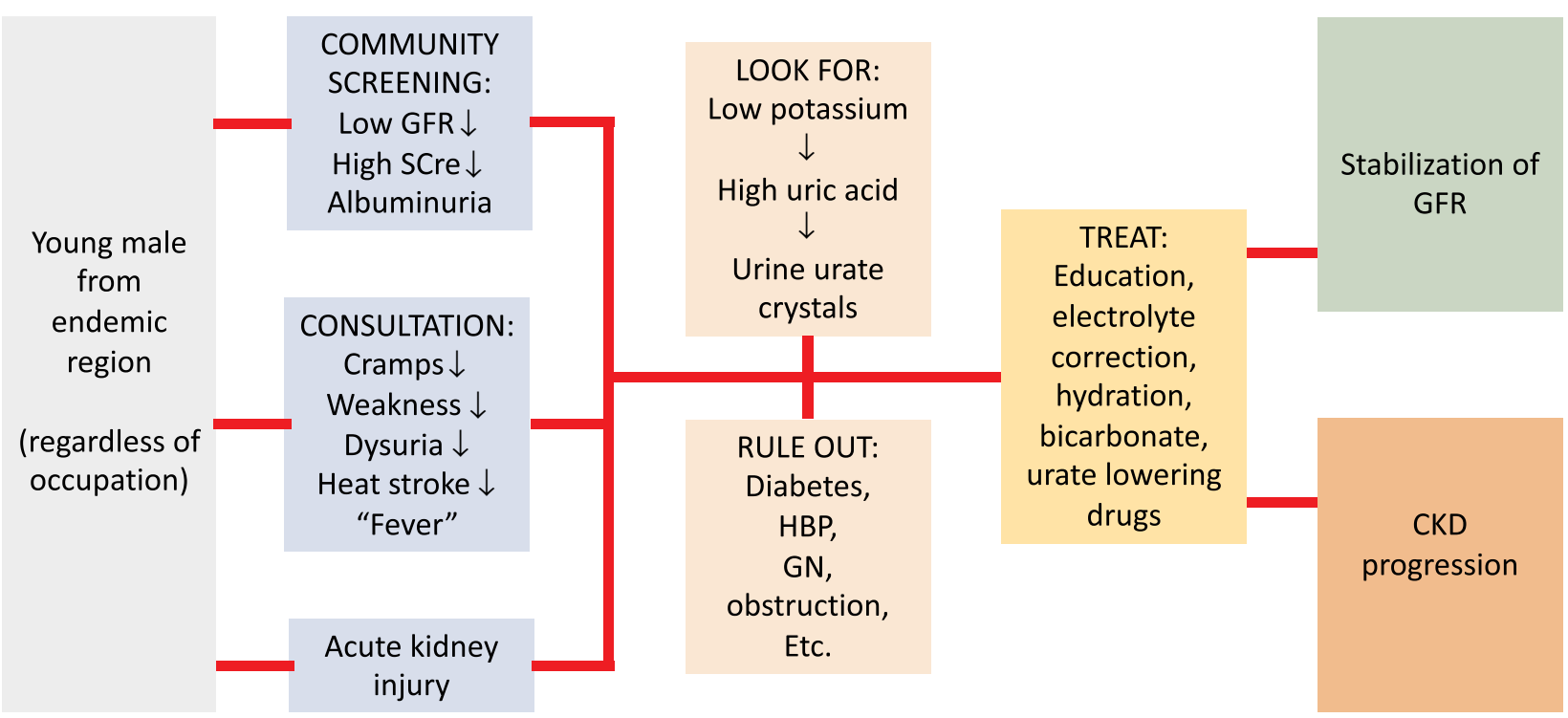

Figure 2 Mesoamerican Nephropathy: Clinical presentation and evolution. From suspected case to progression, the clinical aspects of the management of these patients are similar in all countries of the region.

Abbreviations: V, and/or; GFR, glomerular filtration rate; CKD, chronic kidney disease; GN, glomerulonephritis; HBP, high blood pressure; SCre, serum creatinine.

hypotheses with regards to environmental, occupational and hereditary factors have been postulated. ${ }^{20}$ Recently, 52 epidemiological studies from sugarcane cutters were evaluated showing they are under physical and mental thermal overload conditions, are more exposed to pollutants and to accidents. The main effects observed were respiratory, cardiovascular, kidney, musculoskeletal, heat stress, dehydration, genotoxic, and those due to accidents. ${ }^{30}$ Kidney damage was 2.5 to 4 times more frequent in men than in women, indicative of an occupational etiology. The data leave no doubt that manual laborers, not just agricultural workers, in hot climate are by far the most affected. For instance, in La Paz Centro, Nicaragua, CKD is strongly related to brick workers who are also exposed to heat stress. ${ }^{31}$

This evidence suggests that although everyone can be affected by heat stress, vulnerable populations such as agricultural workers, especially sugar cane cutters, may be at higher risk. In addition to heat stress, intensive physical activity results in salt and volume loss and episodes of recurrent dehydration. At least in experimental studies, an inflammatory cascade is induced by heat stress and exertion, resulting in the activation of fructokinasemediated glucose cycle disturbances, vasopressin, and aldose reductase, which may be further amplified when rehydration with sugary beverages. ${ }^{32}$ Likewise, uric acid has been shown to be an important mediator that generates tubulointerstitial inflammation and progressive loss of tubular and glomerular function, stimulating the genesis and progression of a slow, degenerative and irreversible in

Table I Definition of CKDu

\section{Suspected CKDu}

I. eGFR $<60 \mathrm{~mL} / \mathrm{min} / \mathrm{I} .73 \mathrm{~m}^{2}$.

2. and or albuminuria $>30 \mathrm{mg} / \mathrm{g}$

3. and or proteinuria $>150 \mathrm{mg} / \mathrm{g}$

All Any of the above in the absence of HT, DM, AKI.

Exclusion criteria; proteinuria $>2 \mathrm{~g} / \mathrm{g}$ excludes the diagnosis

\section{Probable CKDu}

I. Fulfill the criteria of "Suspected CKDu"

2. Hypokalemia and/or hyperuricemia

3. I AND 2 in the absence of autoimmune disease, glomerular disease, congenital kidney disease, obstructive kidney disease Confirmed after repeat assessment at week 12

\section{Confirmed CKDu}

All of the above-mentioned criteria for probable CKDu

AND histopathological features consistent with CKDu on kidney biopsy (pyelonephritis and interstitial nephritis caused by specific etiology should be excluded from the definition).

* In those cases where kidney biopsy is not possible, CKDu can be clinically confirmed with the above mentioned criteria for probable CKDu

Note: Adapted from Anupama YJ, Sankarasubbaiyan S, Taduri G. Chronic kidney disease of unknown etiology: case definition for India - a perspective. Indian J Nephrol. $2020 .^{28}$ 


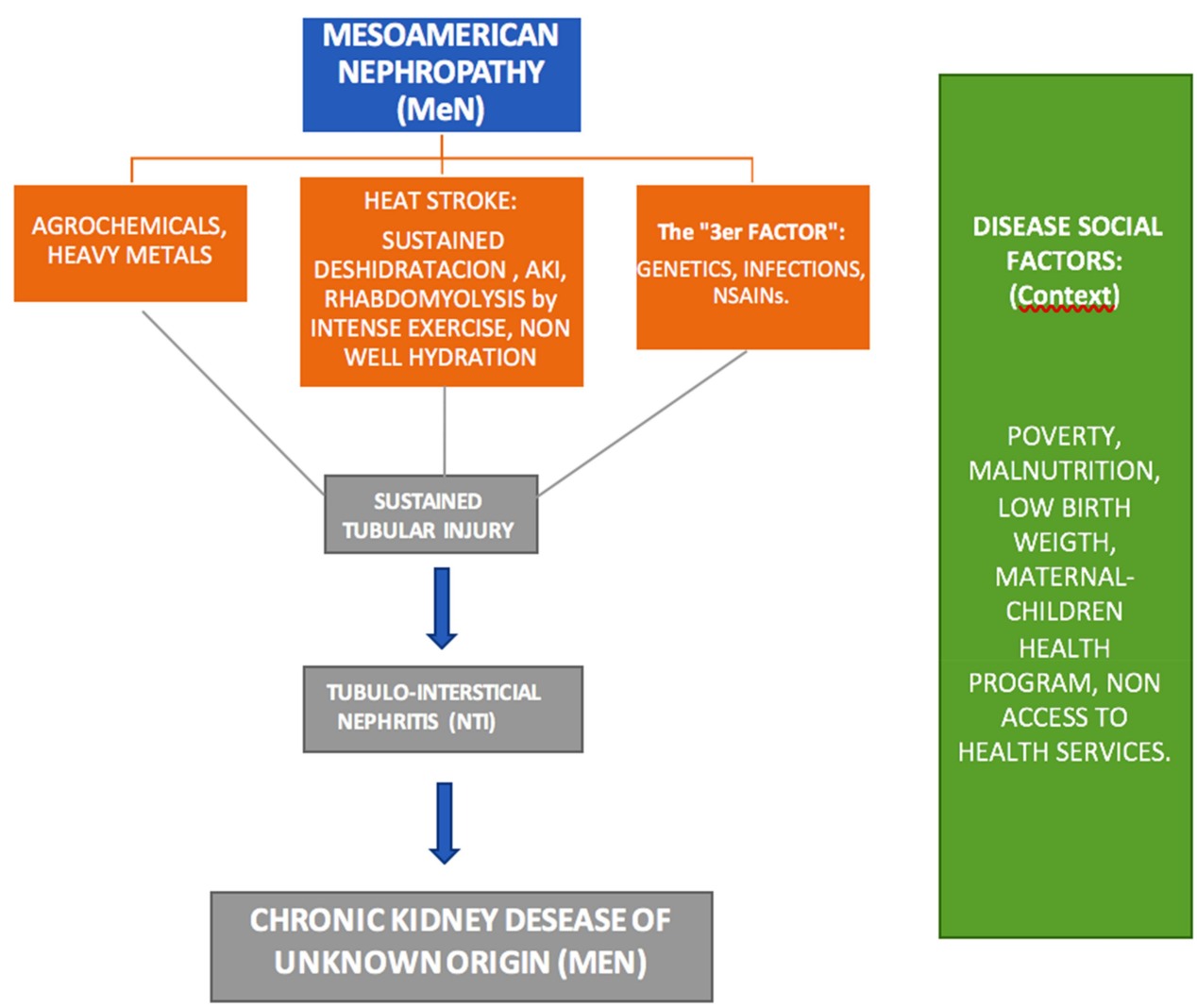

Figure 3 Mesoamerican Nephropathy: Risk factors and pathophysiology. The four elements on which MeN's physiopathology focuses. The community exposed to agrochemicals, heat stress, dehydration and the third factor that could correspond to genetic and epigenetic alterations, appear as a constant and repetitive stimulus that generate tubular damage and accelerate the progression towards CKD. Those elements develop in the context of disease's social factors who accompany the process.

nature CKD. The sustained dehydration within the MeN context may be a risk factor associated with CKD progression. $^{23,33,34}$

\section{Agrochemicals and Heavy Metals}

Agrochemicals and heavy metals have been subject of discussion and analysis in the physiopathology of MeN. Rivers, environment, soil and production land have been thought to be contaminated with these elements. Particularly agrochemicals such as glyphosate, paraquat or heavy metals such as lead, arsenic, cadmium and silica have been associated with the disease; however, as of today, there is no proven causeeffect relationship. Agricultural communities on the southern coast of Guatemala, El Salvador, and Nicaragua are settled on volcanic land, rich in heavy metals. However, several studies have not demonstrated a direct relationship to the genesis, development or progression of $\mathrm{MeN}$ arising from such exposure. ${ }^{23,35,36}$

In a recent study from Nicaragua, elevated levels of aluminum and total arsenic, as well as metabolites of several pesticides, were detected across the population.
However, no differences were identified between the declining and stable kidney function groups against the levels of metals or pesticides tested. ${ }^{37}$ In another recent study of kidney biopsies from agricultural workers, large dysmorphic lysosomes in proximal tubular cells suggested a tubular toxicity mechanism similar to what has been seen with calcineurin inhibitor damage. ${ }^{38}$ Nevertheless, pesticides or heavy metals were not directly measured in any of these subjects and the histopathology findings were recently contested as non-specific. ${ }^{39}$

Silica $(\mathrm{SI}+)$ has recently been described in a North Carolina study as having kidney toxicity. They found a positive relationship between occupational silica exposure and CKD. A dose-response trend of increasing CKD risk with increasing duration of silica exposure was observed and was particularly strong among non-whites. Occupational exposure to silica was associated with a $37 \%$ increased risk of CKD and appeared to be specifically related to unclassified $\mathrm{CKD}$, representing earlier stages of diagnosed CKD. ${ }^{40}$ It should be noted that Silica is one of the particles found on ashes produced by the burning of 
sugar cane, a common practice before workers cut the cane during the harvest season. This ash is so common, it is referred to as the "black snow" in sugar cane regions of Central America. As of today, these theories are only speculative without solid evidence to support them. Finally, a recent study from California demonstrated that areas with high levels of unexplained kidney disease were related to higher average nitrate in groundwater. These findings link kidney disease of unknown origin to farming activities but do not elucidate the etiology of the disease. ${ }^{41}$

\section{The "3rd Factor": Genetics and Epigenetics?}

Different environmental factors in addition to the genetic background may explain differences or similarities between different geographical locations. There are recent observations that direct our attention to genetics and epigenetics. First, none of the proposed local environmental factors appears to be specific to the region or occupational groups developing disease. Second, it is unclear why some individuals develop CKD after minimal exposure to suspected risk factors whereas others remain unaffected after decades of exposure. Third, both anecdotal accounts and published evidence about family history as a risk factor for MeN point to the possibility that some individuals in the region are more susceptible than others. ${ }^{35,42}$ In Figure 3, we describe the currently considered elements of probable physiopathology, adding the possibility of a third factor, accompanied by the social factors of disease.

Some studies on MeN have suggested that a family history of CKD could be a relevant yet unexplored factor, though there is no consensus or definitive evidence about a genetic influence or inheritance of the disease. Many authors rule out this hypothesis firsthand in order to support a fully environmental or occupational cause, such as intense agricultural work or pesticides exposure. Nonetheless, it has been suggested there could be a predisposition to $\mathrm{MeN}$, both in sugar cane workers and in healthy young people. For instance, in sugar cane workers, urinary neutrophil gelatinase-associated lipocalin (NGAL), used as a marker of acute kidney injury, was present and related to loss of kidney function. In the absence of extreme physical work, a genetic predisposition or environmental factor beyond working conditions could be implicated. $^{43-45}$ The recurring finding of biomarkers of kidney injury among children in MeN hotspots could point to a genetic predisposition or to an early-age exposure to a yet-to-be-determined nephrotoxic agent. ${ }^{46}$ Regrettably, the possible influence of hereditary factors was proposed 15 years ago but has not been examined yet and is still an important field for future studies. ${ }^{20}$

There are important gaps to be addressed in the understanding of biological factors and kidney disease. ${ }^{47}$ First, education and awareness of clinicians, researchers, and patients is still limited. This is particularly relevant in terms of translation of research findings into therapeutics, as well as understanding the attributable aspect of genetics to the overall burden of kidney disease. Second, the majority of genetic research is carried out in populations of European ancestry, even though indigenous and other disadvantaged populations have shown higher rates of kidney disease. This lack of diversity substantially hinders the generalizability of genetic findings, raises the issue of potentially misclassifying benign variants as pathogenic, and limits the understanding of kidney disease among disadvantaged populations. ${ }^{48}$ An improved knowledge of genetic and developmental determinants of kidney disease and their interaction with environmental factors may provide important insights into kidney pathophysiology, which in turn could facilitate the development of more effective prevention strategies and pave the way for novel therapeutics.

Importantly, in the context of poverty, physical, environmental and psychological stresses can lead to neurohormonal and/or epigenetic changes that could adversely impact CKD as well as CKD risk factors. In the case of MeN, it is possible that many factors influencing the disease could contribute to epigenetic changes that can in turn influence the expression of $\mathrm{CKD}$ and $\mathrm{CKD}$ risk factors which could accelerate a decline in kidney function (Figure 4).

\section{Others. Use of Nonsteroidal}

\section{Anti-Inflammatory Drugs (NSAIDs) and} Infections

Several studies have demonstrated a frequent use of selfprescription over-the-counter medications among these populations, particularly NSAIDs and other pain killers, which are known causes of AKI and may speed up CKD progression, but there is no evidence that these drugs induce kidney damage in $\mathrm{MeN}^{23}$ With regard to a possible association of $\mathrm{MeN}$ and infectious diseases, it is worth noticing that Malaria was also endemic in the same Pacific Ocean coastal areas, and Hanta Virus and Leptospirosis have also been mentioned, among others. All of these infectious diseases can certainly develop AKI but have not been proven to cause MeN. Further 


\section{Conceptual Model of Socioeconomics Influencing Genetic}

and Epigen etic Changes in CKD-MeN

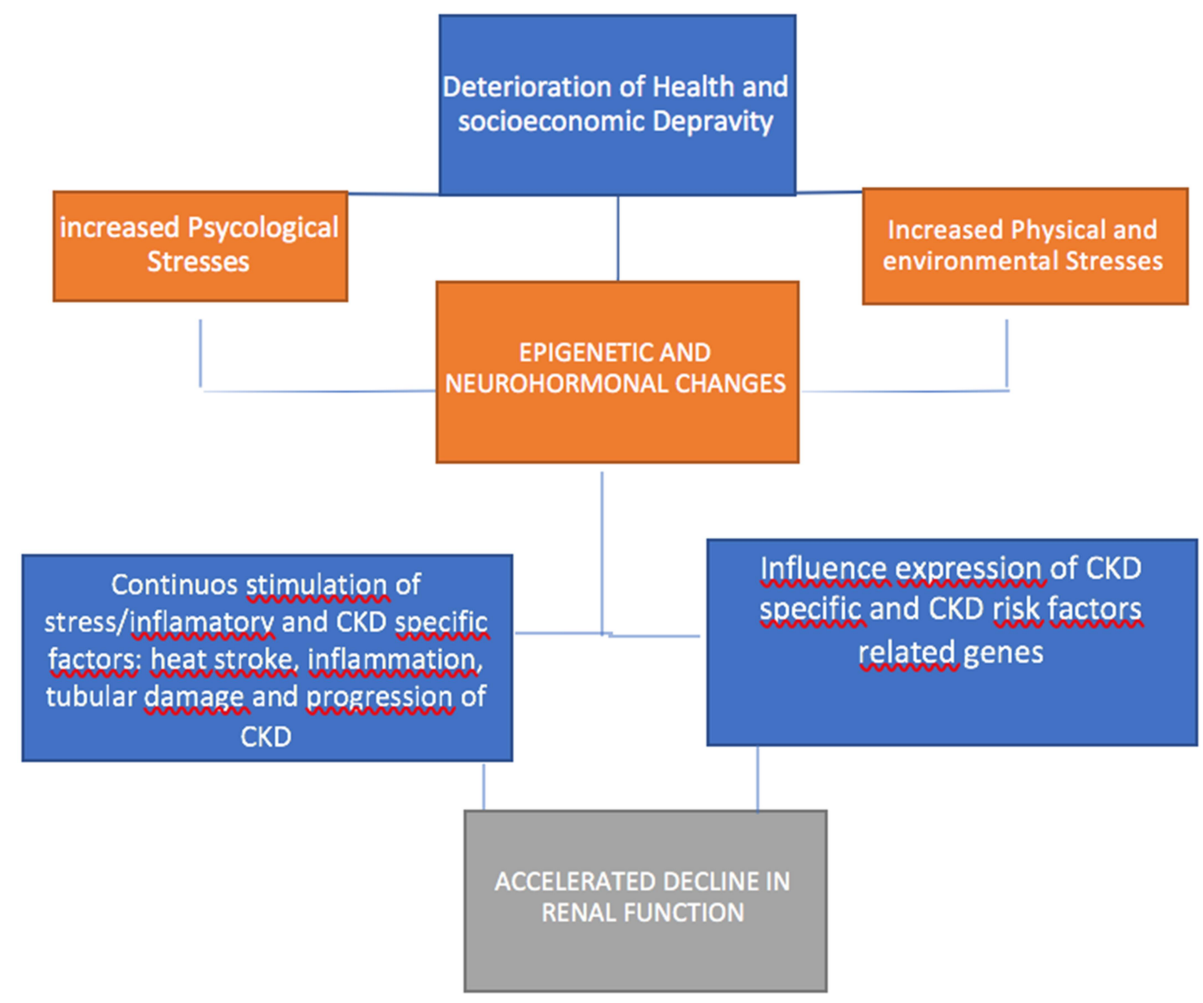

Figure 4 Mesoamerican Nephropathy: Conceptual model. A conceptual model on the influence of social determinants of disease.

studies are needed to demonstrate the relationship between infections and $\mathrm{MeN}^{23}$

\section{Social Determinants of MeN}

During the last decades, it has been increasingly recognized that CKD is more frequent and has suboptimal outcomes among populations or communities considered "disadvantaged". ${ }^{-11,49}$ Agricultural communities in Mesoamerica (Mexico and Central America) live in poverty and/or extreme poverty. The causes of CKD in disadvantaged populations are varied and reflect exposures to environmental, socioeconomic and other factors that contribute to a reduction in nephron mass over time. For instance, low birth weight and nutritional factors, exposure to toxins and infections, may further aggravate damage related to heat stress and dehydration and contribute to the development and progression of CKD. In Guatemala, the situation is dire for the children. Cerón et al describe a significant high incidence of CKD found along the southern coast of Guatemala. They also emphasize paying more attention on diagnosing CKD in children, in order to establish whether there are, in fact, more cases of nontraditional causes, or if diagnoses of undetermined cause are recorded, as it has not been possible to rule out other causes. ${ }^{50}$ These social determinants of the MeN not only serve as background, but rather accompany the communities throughout the CKD process, being an element that does not allow the cycle to close as each time, new generations are exposed to a continuum of chronic, degenerative, progressive and irreversible disease (Figure 5). ${ }^{51}$

\section{Current Prevention and Treatment Options Prevention}

Currently, preventive measures are focused on intervening on the risk factors that have been associated with the development of MeN. ${ }^{23,33}$ When considering the negative impact that thermal stress can have on the kidney, efforts have been made to avoid dehydration and thermal stress to 


\section{THE SCOURGE OF MeN}

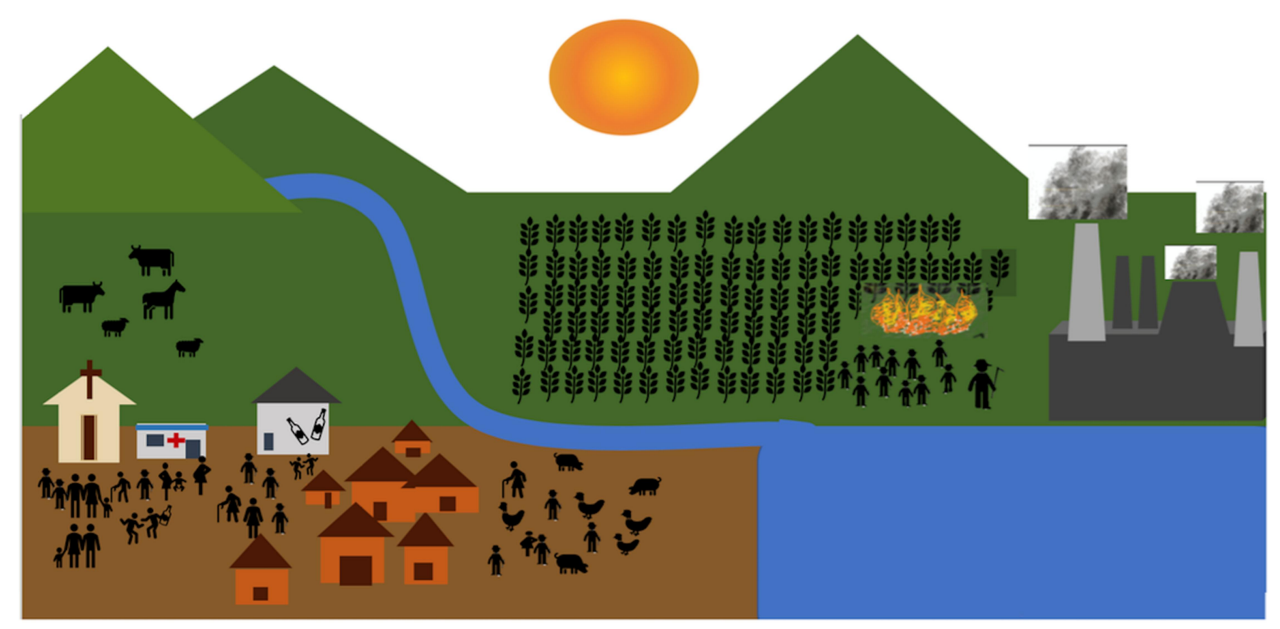

Figure 5 The multicausal phenomenon of Mesoamerican Nephropathy. The community is exposed to social determinants of poverty, genetic and epigenetic changes. It is located at less than 300 meters above sea level and is exposed to heat, contaminated water. However, there is a group of agricultural workers who, in addition, are exposed to heat stress, exercise, dehydration, agrochemicals and Silica particles. All circumstances are given in this group to develop the perfect storm that triggers and accelerates the entire physiopathological process of the $\mathrm{MeN}$.

workers in endemic areas. To prevent heat stress at work, controlled work schedules are sought, facilitate adequate hydration, and develop spaces for shade and rest. ${ }^{52}$ These principles, hydration, shade and rest, have been implemented by some Central American countries to legal frameworks, such is the case of El Salvador with decree 254 in the general law on risk prevention in the workplace. In the same way, Costa Rica has generated different decrees from the Ministry of Health regulating the working conditions of agricultural workers in endemic regions. ${ }^{53}$

The relationship between hydration status and kidney health has been studied for years. ${ }^{54,55}$ In an elegant and well-designed study, Pecoits-Filho et al demonstrated that foundry factory workers in southern Brazil exposed to heat stress had a greater decline in eGFR, changes in blood pressure, serum potassium and uric acid. They are able to demonstrate the negative physiological acute effects in kidney function of indoor heat stress. ${ }^{56}$ Another series of interventional studies showed the feasibility of providing sugar cane workers with adequate access to drinking water, rest and shaded spots. ${ }^{57}$ Although these studies have demonstrated a less incident kidney injury, none of these studies have proven to prevent CKD progression.

Access to proper hydration and rehydration with water and oral rehydration salts is a universal recommendation. Some protocols include providing water in camelbacks so agricultural workers can drink an average of 1 liter/day.
The assessment of adequate hydration in workers can be carried out by measuring body weight before and after a workday, where the decrease in weight after a day translates into the loss of volume. ${ }^{57}$

On the other hand, an early diagnosis is sought by educating workers, screening programs in at-risk populations, and training primary care physicians in the implementation of screenings, diagnosis of kidney disease and their timely referral to the nephrologist. For instance, in Costa Rica, training programs for primary care personnel and the development of a care network that connects the three levels of care have led to an increase in early diagnosis of CKD. The implementation of MeN prevention measures involves the participation of multiple actors from healthy, industry, policy makers and NGOs that actively collaborate in education processes for the population in endemic areas, seeking the development of adequate kidney health prevention and treatment.

\section{Treatment}

Given the lack of compelling evidence, there are no standardized guidelines for the management of $\mathrm{MeN}$ patients. Clinicians in Mesoamerica however have similar approaches to MeN. Some of the common practices from nephrologists in Mesoamerica include the use of allopurinol, potassium supplements, potassium-sparing diuretics, bicarbonate, corticosteroids and sometimes the prescription of angiotensin- 
converting enzyme inhibitors (ACEIs) and angiotensin receptor blockers (ARBs) at low doses. Given the risk for AKI with dehydration and the concomitant use of RAAS blockade, the use of these medications remains a controversial matter. Nephrologists from El Salvador have infrequently used corticosteroids when biopsies demonstrated a lymphocytic infiltrate suggestive of a possible tubulointerstitial nephritis. Mild to moderate hypokalemia, which can be symptomatic and frequently leads to regular visits to the emergency services, is treated with fruit-rich diet and spironolactone, even in advanced CKD. In addition to this treatment, supplemental potassium (potassium gluconate or potassium citrate) can be added. Allopurinol has been found in animal models to have a kidney protective effect against heat stress and is used widely in the region. Sodium bicarbonate delays the progression of chronic kidney disease, ${ }^{58-60}$ and also in rats has showed a protective role to prevent kidney injury from recurrent heat stress. ${ }^{61}$ Although there is no clinical evidence for the use of allopurinol and bicarbonate, we use it empirically in MeN patients with caution, while more evidence is available.

We also recommend avoiding sugary drinks, alcoholic drinks, and nephrotoxic drugs, mainly NSAIDs and aminoglycosides. We discourage the use of diuretics, widely used for the treatment of other edema-inducing CKDs but counterproductive in these patients with dehydration and electrolyte disturbances.

The CENCAM Third International Workshop on CKDu, held in San Jose, Costa Rica in March 2019 made several recommendations that are summarized in Table 2.

Government policies should focus on occupational health and safety protections, particularly in professions with high levels of thermal exposure; basic services provision with a focus on safe and adequate drinking water; and development of healthcare infrastructure to increase access to medical care in CKDu-affected regions and to establish disease management protocols and registries.

Overall, for the general management of $\mathrm{MeN}$, we recommend:

1. Establish systematic kidney disease data registries and surveillance programs, specifically for $\mathrm{MeN}$ or incorporated into broader CKD programs.

2. Create and enforce laws protecting against unsafe working conditions, particularly conditions exposing individuals to undue thermal stress, harsh labor, inappropriate hydration practices, and inadequate protection against agrochemical products.
Table 2 Recommendations for Clinical Management of Patients with CKDu by Healthcare Providers

I. Ensure adequate access to safe drinking water and promotion of appropriate hydration.

2. Encourage pursuing frequent rest breaks and reducing heat exposure in the occupational setting (rest and shade).

3. Emphasize avoidance of nephrotoxic medications, especially NSAIDs and over the counter medications not requiring oversight by a healthcare provider

4. Emphasize avoidance of all herbal medications

5. Encourage minimization of alcohol consumption, with a focus on avoidance of home-brewed alcohol

6. Counsel on using appropriate personal protective equipment and avoiding agrichemical exposures

7. Establish regular care with physicians, ideally nephrologists, who can diagnose early the presence of kidney dysfunction and manage sequelae of chronic kidney disease including electrolyte and acid-base disturbances

8. There is insufficient evidence at present to support the use of pharmacotherapy to prevent progressive kidney function decline in CKDu, including ACEls and ARBs, allopurinol, and corticosteroids. However, use of these and other medications in appropriate clinical situations arising concurrently with CKDu may be appropriate for some patients.

Note: Clinical Considerations surrounding Chronic Kidney Disease of Undetermined Etiology (CKDu): A report from the Third International Workshop of CKDu, San Jose, CR, March 2019.62

3. Develop infrastructure focused on the provision of safe, clean, and widely available drinking water.

4. Train more nephrologists and general physicians and provide additional training to other healthcare and social service providers in the specifics of caring for MeN.

5. Expand the medical infrastructure needed to care for MeN patients, particularly facilities that can conduct kidney biopsies; expand and modernize the capacity for KRT provision.

6. Develop educational campaigns to expand awareness and recognition of $\mathrm{MeN}$, fighting fear and misinformation.

7. Engage with affected individuals and communities to understand their priorities regarding the management of MeN.

\section{Summary}

$\mathrm{MeN}$ is now recognized as a public health problem in the Mesoamerican region. Disadvantaged populations overrepresent the disease and proper access to prevention and 
treatment has been limited or nonexistent to this population. Multiple hypotheses for its etiology such as heat stress, agrochemicals and pesticides, genetics and other risk factors have been proposed. As of today, no intervention has been proven to be successful in preventing the disease progression. Until more evidence is available, we provide our knowledge and experience for the current management of the disease in the region. There are many research opportunities and priorities that should include clinical trials to evaluate the efficacy and safety of the current treatment protocols, along with etiological and genetic studies, and the development of kidney disease data systems which will aid our current data blindness. Local resources and funding have been historically limited, and the burden of clinical work is high. Research priorities should include longitudinal studies, provisions for biorepositories, reinforcing the capacity for kidney biopsies, diagnosis and investigation of pathogenesis in addition to an open access database. ${ }^{27}$ Given the complexity and systemic nature of this problem, the required interventions are interdisciplinary and multidimensional. Therefore, it is not only a matter incumbent to the medical community. Only global scale efforts involving multiple stakeholders will be able to close the observed gaps in kidney care that disadvantaged populations experience. The World Health Organization (WHO) has established sustainable health goals that are only possible with a multidisciplinary approach. Our regional nephrologists and societies urge the support and foster of the international health and kidney organizations with a stronger and direct engagement. Finally, in fairness to patients and their communities, we encourage any research partnership should also contribute as much as possible to strengthen local capacities, train human resources and empower the vulnerable populations suffering the disease.

\section{Disclosure}

The authors report no conflicts of interest in this work.

\section{References}

1. Almaguer M, Herrera R, Orantes CM. Chronic kidney disease of unknown etiology in agricultural communities. MEDICC Rev. 2014;16(2):9-15.

2. Remuzzi G, Perico N. International society of nephrology's perspective on the emergence of chronic kidney diseases of unknown/undetermined etiology. MEDICC Rev. 2014;16(2):75-76.

3. Wanigasuriya KP, Peiris-John RJ, Wickremasinghe R, Hittarage A. Chronic renal failure in North Central Province of Sri Lanka: an environmentally induced disease. Trans $R$ Soc Trop Med Hyg. 2007;101(10):1013-1017. doi:10.1016/j.trstmh.2007.05.006
4. Ganguli A. Uddanam nephropathy/regional nephropathy in India: preliminary findings and a plea for further research. Am J Kidney Dis. 2016;68(3):344-348. doi:10.1053/j.ajkd.2016.04.012

5. Wijkström J, Leiva R, Elinder CG, et al. Clinical and pathological characterization of Mesoamerican nephropathy: a new kidney disease in Central America. Am J Kidney Dis. 2013;62(5):908-918. doi:10.1053/j.ajkd.2013.05.019

6. Jayasumana C, Orantes $\mathrm{C}$, Herrera R, et al. Chronic interstitial nephritis in agricultural communities: a worldwide epidemic with social, occupational and environmental determinants. Nephrol Dial Transplant. 2017;32(2):234-241. doi:10.1093/ndt/gfw346

7. Cohen J. Mesoamerica's mystery killer. Science. 2014;344 (6180):143-147. doi:10.1126/science.344.6180.143

8. Correa-Rotter R, Wesseling C, Johnson RJ. CKD of unknown origin in Central America: the case for a Mesoamerican nephropathy. Am J Kidney Dis. 2014;63(3):506-520. doi:10.1053/ j.ajkd.2013.10.062

9. Coresh J, Jafar TH. Disparities in worldwide treatment of kidney failure. Lancet. 2015;385(9981):1926-1928. doi:10.1016/S01406736(14)61890-0

10. Ene-Iordache B, Perico N, Bikbov B, et al. Chronic kidney disease and cardiovascular risk in six regions of the world (ISN-KDDC): a cross-sectional study. Lancet Glob Health. 2016;4(5):e307-e319. doi:10.1016/S2214-109X(16)00071-1

11. Liyanage $T$, Ninomiya $T$, Jha $V$, et al. Worldwide access to treatment for end-stage kidney disease: a systematic review. Lancet. 2015;385 (9981):1975-1982. doi:10.1016/S0140-6736(14)61601-9

12. Disadvantaged populations [Internet]. Available from: https://www. theisn.org/topics/disadvantaged-populations. Accessed July 1st, 2020

13. Obrador GT, Rubilar X, Agazzi E, Estefan J. The challenge of providing renal replacement therapy in developing countries: the Latin American perspective. Am J Kidney Dis off J Natl Kidney Found. 2016;67(3):499-506. doi:10.1053/j.ajkd.2015.08.033

14. García-Trabanino R, Hernández C, Rosa A, Domínguez Alonso J, on behalf of the Emergency Social Fund for Health (FSES) of Tierra Blanca, Usulután, El Salvador. Incidence, mortality, and prevalence of end-stage chronic renal disease in the Bajo Lempa region of El Salvador: a ten-year community registry. Nefrologia. 2016;36 (5):517-522. doi:10.1016/j.nefro.2016.03.018

15. Garcia-Trabanino R, Aguilar R, Reyes Silva C, Mercado MO, Merino RL. End-stage renal disease among patients in a referral hospital in El Salvador. Rev Panam Salud Publica. 2002;12:202-206. doi:10.1590/s1020-49892002000900009

16. Brooks D Final scoping study report: epidemiology of chronic kidney disease in Nicaragua [report] Boston University; 2009. Available from: http://www. cao-ombudsman.org/cases/documentlinks/documents/03H_BU_FINAL_ report_scopestudyCRI_18.Dec.2009.pdf. Accessed July 1st, 2020.

17. Wesseling C, van Wendel de Joode B, Crowe J, et al. Mesoamerican nephropathy: geographical distribution and time trends of chronic kidney disease mortality between 1970 and 2012 in Costa Rica. Occup Environ Med. 2015;72:714-721. doi:10.1136/oemed-2014102799

18. Domínguez J, Moya-Pérez C, Jansá JM. Análisis de prevalencia y determinantes de la insuficiencia renal crónica en la costa del Océano Pacífico: sur de México, Guatemala, El Salvador y Honduras. Agencia Municipal de Salud Pública, Barcelona, Catalunya, España. In: SALTRA, editor. Chronic Kidney Disease: Assessment of Current Knowledge and Feasibility for Regional Research Collaboration in Central America, Section 1. Vol. 2. 1st. Heredia, Costa Rica: Salud y Trabajo en América Central; 2006:23-24

19. Cerdas M. Chronic kidney disease in Costa Rica. Kidney Int Suppl. 2005;97:S31-S33. doi:10.1111/j.1523-1755.2005.09705.x

20. García-Trabanino R, Domínguez J, Jansà JM, Oliver A. Proteinuria and chronic renal failure in the coast of El Salvador: detection with low cost methods and associated factors. Nefrologia. 2005;25 (1):31-38. 
21. Pan American Health Organization. Chronic Kidney Disease in Agricultural Communities in Central America: Concept Paper. Washington, D.C.: PAHO; 2013. Available from: http://www.paho. $\mathrm{org} / \mathrm{hq} / \mathrm{index} . \mathrm{php}$ ?option $=$ com_docman\&task $=$ doc_download\&gid $=$ 22051\&Itemid=270\&lang=en. Accessed July 1st, 2020.

22. Fischer RSB, Mandayam S, Chavarria D, et al. Clinical evidence of acute Mesoamerican nephropathy. Am $J$ Trop Med Hyg. 2017;97:1247-1256. doi:10.4269/ajtmh.17-0260

23. Correa-Rotter R, Garcia-Trabanino R. Mesoamerican nephropathy. Semin Nephrol. 2019;39(3):263-271. doi:10.1016/j.semnephrol.2019.02.004

24. Ferreiro A, Alvarez-Estevez G, Cerdas-Calderon M, et al. Confirmed clinical case of chronic kidney disease of nontraditional causes in agricultural communities in Central America: a case definition for surveillance. Rev Panam Salud Publica. 2016;40:301-308.

25. Gonzalez-Quiroz M, Smpokou ET, Silverwood RJ, et al. Decline in kidney function among apparently healthy young adults at risk of mesoamerican nephropathy. $J$ Am Soc Nephrol. 2018;29 (8):2200-2212. doi:10.1681/ASN.2018020151

26. Fischer RSB, Vangala C, Mandayam S, et al. Clinical markers to predict progression from acute to chronic kidney disease in Mesoamerican nephropathy. Kidney Int. 2018;94(6):1205-1216. doi:10.1016/j.kint.2018.08.020

27. Caplin B, Yang CW, Anand S, et al. The International Society of Nephrology's International Consortium of Collaborators on chronic kidney disease of unknown etiology: report of the working group on approaches to population-level detection strategies and recommendations for a minimum dataset [published correction appears in Kidney Int. 2019 Apr;95(4):997-998]. Kidney Int. 2019;95(1):4-10. doi:10.1016/j.kint.2018.08.019

28. Anupama YJ, Sankarasubbaiyan S, Taduri G. Chronic kidney disease of unknown etiology: case definition for India - a perspective. Indian J Nephrol. 2020.

29. Aguilar-Ramirez D, Raña-Custodio A, Villa A, et al. Decreased kidney function and agricultural work: a cross-sectional study in middle-aged adults from Tierra Blanca, Mexico [published online ahead of print, 2020 May 22]. Nephrol Dial Transplant. 2020; gfaa041. doi:10.1093/ndt/gfaa041

30. Leite MR, Zanetta DMT, Trevisan IB, Burdmann EA, Santos UP. Sugarcane cutting work, risks, and health effects: a literature review. Rev Saude Publica. 2018;52:80. doi:10.11606/s1518-8787.2018052000138

31. Gallo-Ruiz L, Sennett CM, Sánchez-Delgado M, et al. Prevalence and risk factors for CKD among brickmaking workers in $\mathrm{La} \mathrm{Paz}$ Centro, Nicaragua. Am J Kidney Dis. 2019;74(2):239-247. doi:10.1053/j.ajkd.2019.01.017

32. García-Arroyo FE, Cristóbal M, Arellano-Buendía AS, et al. Rehydration with soft drink-like beverages exacerbates dehydration and worsens dehydration-associated renal injury. Am J Physiol Regul Integr Comp Physiol. 2016;311(1):R57-R65. doi:10.1152/ajpregu.00354.2015

33. Johnson RJ, Wesseling C, Newman LS. Chronic kidney disease of unknown cause in agricultural communities. N Engl J Med. 2019;381 (7):689. doi:10.1056/NEJMc1907676

34. Roncal-Jimenez C, García-Trabanino R, Barregard L, et al. Heat stress nephropathy from exercise-induced uric acid crystalluria: a perspective on mesoamerican nephropathy. Am J Kidney Dis. 2016;67(1):20-30. doi:10.1053/j.ajkd.2015.08.021

35. González-Quiroz M, Pearce N, Caplin B, Nitsch D. What do epidemiological studies tell us about chronic kidney disease of undetermined cause in Meso-America? A systematic review and meta-analysis. Clin Kidney J. 2018;11(4):496-506. doi:10.1093/ckj/sfx136

36. Valcke M, Levasseur M, Soares da Silva A, et al. Pesticide exposures and chronic kidney disease of unknown etiology: an epidemiologic review. Environ Health. 2017;16(49). doi:10.1186/s12940-017-0254-0

37. Smpokou ET, González-Quiroz M, Martins C, et al. Environmental exposures in young adults with declining kidney function in a population at risk of Mesoamerican nephropathy. Occup Environ Med. 2019;76(12):920-926. doi:10.1136/oemed-2019-105772
38. Vervaet BA, Nast CC, Jayasumana C, et al. Chronic interstitial nephritis in agricultural communities is a toxin-induced proximal tubular nephropathy. Kidney Int. 2020;97(2):350-369. doi:10.1016/j. kint.2019.11.009

39. Wijkström J, Elinder CG, Hultenby K, Söderberg M, Wernerson A. "Dysmorphic" lysosomes in proximal tubular cells are not specific for CINAC/CKDu and do not provide evidence that CINAC/CKDu is a toxininduced disease [published online ahead of print, 2020 Jun 18]. Kidney Int. 2020;S0085-2538(20)30686-4. doi:10.1016/j.kint.2020.04.057

40. Vupputuri S, Parks CG, Nylander-French LA, Owen-Smith A, Hogan SL, Sandler DP. Occupational silica exposure and chronic kidney disease. Ren Fail. 2012;34(1):40-46. doi:10.3109/ 0886022X.2011.623496

41. Anand S, Staniec A, Montez-Rath M, Vlahos P. Using GIS mapping to track hot spots of kidney disease in California. $N$ Engl $J$ Med. 2020;382(23):2265-2267. doi:10.1056/NEJMc2001023

42. Nicholas SB, Kalantar-Zadeh K, Norris KC. Socioeconomic disparities in chronic kidney disease. Adv Chronic Kidney Dis. 2015;22 (1):6-15. doi:10.1053/j.ackd.2014.07.002

43. Castillo-Rodriguez E, Fernandez-Prado R, Martin-Cleary C, et al. Kidney injury marker 1 and neutrophil gelatinase-associated lipocalin in chronic kidney disease. Nephron. 2017;136(4):263-267. doi: $10.1159 / 000447649$

44. Laws RL, Brooks DR, Amador JJ, et al. Biomarkers of kidney injury among nicaraguan sugarcane workers. Am J Kidney Dis. 2016;67 (2):209-217. doi:10.1053/j.ajkd.2015.08.022

45. Ramírez-Rubio O, Amador JJ, Kaufman JS, et al. Urine biomarkers of kidney injury among adolescents in Nicaragua, a region affected by an epidemic of chronic kidney disease of unknown aetiology. Nephrol Dial Transplant. 2016;31(3):424-432. doi:10.1093/ndt/ gfv292

46. Leibler JH, Ramirez-Rubio O, Velázquez JJA, et al. Biomarkers of kidney injury among children in a high-risk region for chronic kidney disease of uncertain etiology [published online ahead of print, 2020 Jun 5]. Pediatr Nephrol. 2020. doi:10.1007/s00467-020-04595-3

47. Obrador GT, Schultheiss UT, Kretzler M, et al. Genetic and environmental risk factors for chronic kidney disease. Kidney Int Suppl (2011). 2017;7(2):88-106. doi:10.1016/j.kisu.2017.07.004

48. Manrai AK, Funke BH, Rehm HL, et al. Genetic misdiagnoses and the potential for health disparities. $N$ Engl J Med. 2016;375 (7):655-665. doi:10.1056/NEJMsa1507092

49. Barsoum RS. Chronic kidney disease in the developing world. $N$ Engl $J$ Med. 2006;354(10):997-999. doi:10.1056/NEJMp058318

50. Cerón A, Fort MP, Morine CM, Lou-Meda R. Chronic kidney disease among children in Guatemala. Rev Panam Salud Publica. 2014;36 (6):376-382.

51. Friedman D, Luyckx VA. Genetic and developmental factors in chronic kidney disease hotspots. Semin Nephrol. 2019;39 (3):244-255. doi:10.1016/j.semnephrol.2019.02.002

52. Wegman DH, Apelqvist J, Bottai M, et al. Intervention to diminish dehydration and kidney damage among sugarcane workers. Scand $J$ Work Environ Health. 2018;44:16-24. doi:10.5271/sjweh.3659

53. OSHA recommendations for occupational heat esposures. US Department of Labor. Available from: https://www.osha.gov/SLTC/ heatstress/. Accessed August 27, 2020.

54. Roncal-Jimenez C, Lanaspa MA, Jensen T, Sanchez-Lozada LG, Johnson RJ. Mechanisms by which dehydration may lead to chronic kidney disease. Ann Nutr Metab. 2015;66(Suppl 3):10-13. doi:10.1159/000381239

55. Roncal-Jimenez CA, García-Trabanino R, Wesseling C, Johnson RJ. Mesoamerican nephropathy or global warming nephropathy? Blood Purif. 2016;41(1-3):135-138. doi:10.1159/000441265

56. Nerbass FB, Moist L, Clark WF, Vieira MA, Pecoits-Filho R. Hydration status and kidney health of factory workers exposed to heat stress: a Pilot Feasibility Study. Ann Nutr Metab. 2019;74(Suppl 3):30-37. doi:10.1159/000500373 
57. García-Trabanino R, Jarquín E, Wesseling C, et al. Heat stress, dehydration, and kidney function in sugarcane cutters in El Salvador-a cross-shift study of workers at risk of Mesoamerican nephropathy. Environ Res. 2015;142:746-755. doi:10.1016/j. envres.2015.07.007

58. Roncal-Jiménez C, Sato Y, Milagres T, et al. Experimental heat stress nephropathy and liver injury are improved by allopurinol. $\mathrm{Am}$ J Physiol Renal Physiol. 2018;315(3):726-733. doi:10.1152/ ajprenal.00543.2017

59. Di Lorio B, Bellasi A, Raphael K, et al. Treatment of metabolic acidosis with sodium bicarbonate delays progression of chronic kidney disease: the UBI Study. J Nephrol. 2019;32(6):989-1001. doi:10.1007/s40620-019-00656-5
60. Goraya N, Wesson DE. Clinical evidence that treatment of metabolic acidosis slows the progression of chronic kidney disease. Curr Opin Nephrol Hypertens. 2019;28(3):267-277. doi:10.1097/ MNH.000000000000049

61. Sánchez-Lozada L, García-Arroyo F, Gonzaga G, et al. Kidney injury from recurrent heat stress and rhabdomyolysis: protective role of allopurinol and sodium bicarbonate. Am J Nephrol. 2018;48:339-348. doi:10.1159/000494663

62. Crowe JL, Joubert BR, Brooks DR, Eds. Report From the Third International Workshop on Chronic Kidney Diseases of Uncertain/ Non-Traditional Etiology in Mesoamerica and Other Regions, SALTRA, 20 March - 22 March 2019, San José, Costa Rica

\section{Publish your work in this journal}

The International Journal of Nephrology and Renovascular Disease is an international, peer-reviewed open-access journal focusing on the pathophysiology of the kidney and vascular supply. Epidemiology, screening, diagnosis, and treatment interventions are covered as well as basic science, biochemical and immunological studies. The manuscript management system is completely online and includes a very quick and fair peer-review system, which is all easy to use. Visit http://www.dovepress.com/testimonials.php to read real quotes from published authors. 